\title{
Deciphering metabolic traits of the fungal pathogen Aspergillus fumigatus: redundancy vs. essentiality
}

\author{
Jorge Amich ${ }^{1}$ and Sven Krappmann ${ }^{1,2} *$ \\ ${ }^{1}$ Research Center for Infectious Diseases, Julius-Maximilians-Universität Würzburg, Würzburg, Germany \\ ${ }^{2}$ Microbiology Institute - Clinical Microbiology, Immunology and Hygiene, University Hospital of Erlangen, Friedrich-Alexander-Universität \\ Erlangen-Nürnberg, Erlangen, Germany
}

\section{Edited by:}

Elaine M. Bignell, Imperial College

London, UK

\section{Reviewed by:}

Bernhard Hube, Leibniz Institute for Natural Product Research and

Infection Biology, Germany

Jean-Paul Latgé, Institut Pasteur,

France

\section{${ }^{*}$ Correspondence:}

Sven Krappmann, Microbiology Institute - Clinical Microbiology,

Immunology and Hygiene, University Hospital of Erlangen,

Friedrich-Alexander-Universität Erlangen-Nürnberg, Wasserturmstr. 3/5, D-91054 Erlangen, Germany. e-mail:sven.krappmann@ uk-erlangen.de
Incidence rates of infections caused by environmental opportunistic fungi have risen over recent decades. Aspergillus species have emerged as serious threat for the immunecompromised, and detailed knowledge about virulence-determining traits is crucial for drug target identification. As a prime saprobe, A. fumigatus has evolved to efficiently adapt to various stresses and to sustain nutritional supply by osmotrophy, which is characterized by extracellular substrate digestion followed by efficient uptake of breakdown products that are then fed into the fungal primary metabolism. These intrinsic metabolic features are believed to be related with its virulence ability. The plethora of genes that encode underlying effectors has hampered their in-depth analysis with respect to pathogenesis. Recent developments in Aspergillus molecular biology allow conditional gene expression or comprehensive targeting of gene families to cope with redundancy. Furthermore, identification of essential genes that are intrinsically connected to virulence opens accurate perspectives for novel targets in antifungal therapy.

Keywords: aspergillosis, nutrients, gene family targeting, conditional promoter replacement, virulence

\section{FUNGI AS OPPORTUNISTIC PATHOGENS}

Pathogenic fungi have been classified historically in two major subgroups: primary and opportunistic ones (van Burik and Magee, 2001). While the former embraces fungi such as Coccidioides immitis or Histoplasma capsulatum that are capable of infecting a healthy host to cause severe systemic diseases, rely opportunistic fungi on an impaired host immune system to establish infection. Environmental saprobes, such as Aspergillus fumigatus or Cryptococcus neoformans are prominent examples from this group and rarely cause disease in a healthy individual. However, the number of associated clinical cases has significantly increased over the last decades, mainly based on modern medicine practices multiplying the number of immunosuppressed, susceptible individuals. Opportunistic fungi usually cannot thrive within the tissue of an immunocompetent individual. This suggests that they do not possess defined and unique characteristics to cause disease, features that have classically been coined as virulence factors (Casadevall and Pirofski, 1999). In fact, virulence of the two major fungal pathogens that are responsible for the majority of opportunistic infections, Candida albicans and A. fumigatus, appears to be multifactorial (Cutler, 1991; Latgé, 2001; Hube, 2004; Abad et al., 2010). This hampers the identification of specific targets against which chemotherapeutic compounds may be active. And indeed, current antifungal chemotherapy, which is mainly based on disrupting the integrity of the fungal cell membrane or wall (Hoehamer et al., 2010), is not satisfactory efficient.

It has become evident that nutritional versatility, uptake systems, and metabolic pathways employed by opportunistic fungi during infection represent fundamental aspects of their pathogenicity. Albeit considered as unspecific virulence determinants, such aspects may constitute novel targets for antifungal therapy. For instance, iron acquisition from host tissues has been proven to be important for virulence of several pathogens, among them fungi as C. neoformans, C. albicans, or A. fumigatus (Ramanan and Wang, 2000; Jung et al., 2008; Haas, 2012). In the latter, in vivo iron acquisition was shown to be dependent on the siderophore system and independent of the iron reductive assimilation pathway (Schrettl et al., 2004), establishing this process as a virulence attribute. This exemplifies how a deeper knowledge of the metabolic status and nutrient acquisition strategies within infected tissues sheds light on fungal virulence mechanisms to assist in identifying novel targets of antifungal therapy (Brock, 2009).

\section{THE HOST REPRESENTS A DISTINCT ENVIRONMENT DURING ASPERGILLOSIS}

Invasive pulmonary aspergillosis (IPA) is a severe infection caused by Aspergillus species (Latgé, 1999) and affects primarily warmblooded animals and as well as immunocompromised humans. Due to the success of immunosuppressive therapies in modern medicine has this disease had a steadily rising incidence during the last decades (McNeil et al., 2001). IPA is characterized by high mortality rates that may reach up to $90 \%$ depending on the host's immune status (Singh and Paterson, 2005; Del Bono et al., 2008; Mikulska et al., 2009). Reasons for this are poor diagnostic means, the inefficiency of current antimycotic regimens (Baddley et al., 2010), and the general severness of the underlying diseases with their consequential risk factors. Among all Aspergillus 
species, A. fumigatus accounts for approximately $90 \%$ of all cases of aspergillosis (Brakhage and Langfelder, 2002). Publication of its genome sequence (Nierman et al., 2005) prompted researchers to look for relevant characteristics and specific genes that could explain this prominent virulence capacity. However, comparative genome analyses failed to identify such specific virulence factors (Tekaia and Latgé, 2005).

It has become more evident that its metabolic versatility enables A. fumigatus to thrive within the surrounding, infected tissues (Rhodes, 2006). In the course of pulmonary infection, A. fumigatus exploits the lung as principal metabolic environment and source of nutriments (Figure 1). In this respect, two major aspects are of special interest to understand the ability of this fungal pathogen to propagate in such a challenging environment: (i) the metabolic status of the fungus in vivo and regulatory cascades, metabolic processes, and stress responses that the fungus depends on within the surrounding tissue matrix, and (ii) the nutritional sources that the fungus exploits within such a highly specific growth medium, limiting nutrients, and the fungal anabolism. The following sections of this mini review will briefly summarize the current knowledge on nutrient acquisition and metabolic adaptation of A. fumigatus during invasive aspergillosis. Furthermore, it will outline approaches to overcome the negative consequences resulting from the pronounced redundancy of gene functions expressed by $A$. fumigatus, which complicates identification of novel antifungal drug targets.

\section{ADAPTING TO THE HOST}

In its natural environment, the soil, A. fumigatus represents a prime saprobe that is characterized by its metabolic versatility and great adaptability. It has been suggested that such a competitive environment has served as evolutionary virulence school for this fungal pathogen (Askew, 2008; Fuller et al., 2011). This implies that $A$. fumigatus expresses distinct features supporting growth in human tissues based on adaptation to the adverse and variable conditions commonly encountered in the wild. Among these, its pronounced resistance to stressful conditions has to be taken into account. Reflecting elevated temperatures present in composting substrates, A. fumigatus is well adapted to temperature stress and displays an exquisite thermotolerance of up to $70^{\circ} \mathrm{C}$ (Bhabhra and Askew, 2005). Moreover, it is well adapted to grow at $37^{\circ} \mathrm{C}$, the actual body temperature of humans, which represents an essential characteristic for pathogenicity. As a further example, hypoxia, which is commonly developed within compost piles (Wang et al., 2007), also develops during IPA in mice (Grahl et al., 2011) and, accordingly, A. fumigatus' adaptation to hypoxic conditions is crucial for virulence (Willger et al., 2008).

In agreement with the importance of adaptation to the host, several stress response pathways and signal transduction cascades of A. fumigatus have been functionally characterized and their contributions to virulence were assessed (see review by Hartmann etal., 2011a). In essence, conditions of thermal stress, alkaline $\mathrm{pH}$, hypoxia, iron depletion, and nitrogen starvation are encountered by the fungal pathogen when infecting a susceptible host. The ladder aspect became evidently clear from in vivo transcriptional profiling data that monitored the host adaptation process of $A$. fumigatus during early infection of neutropenic

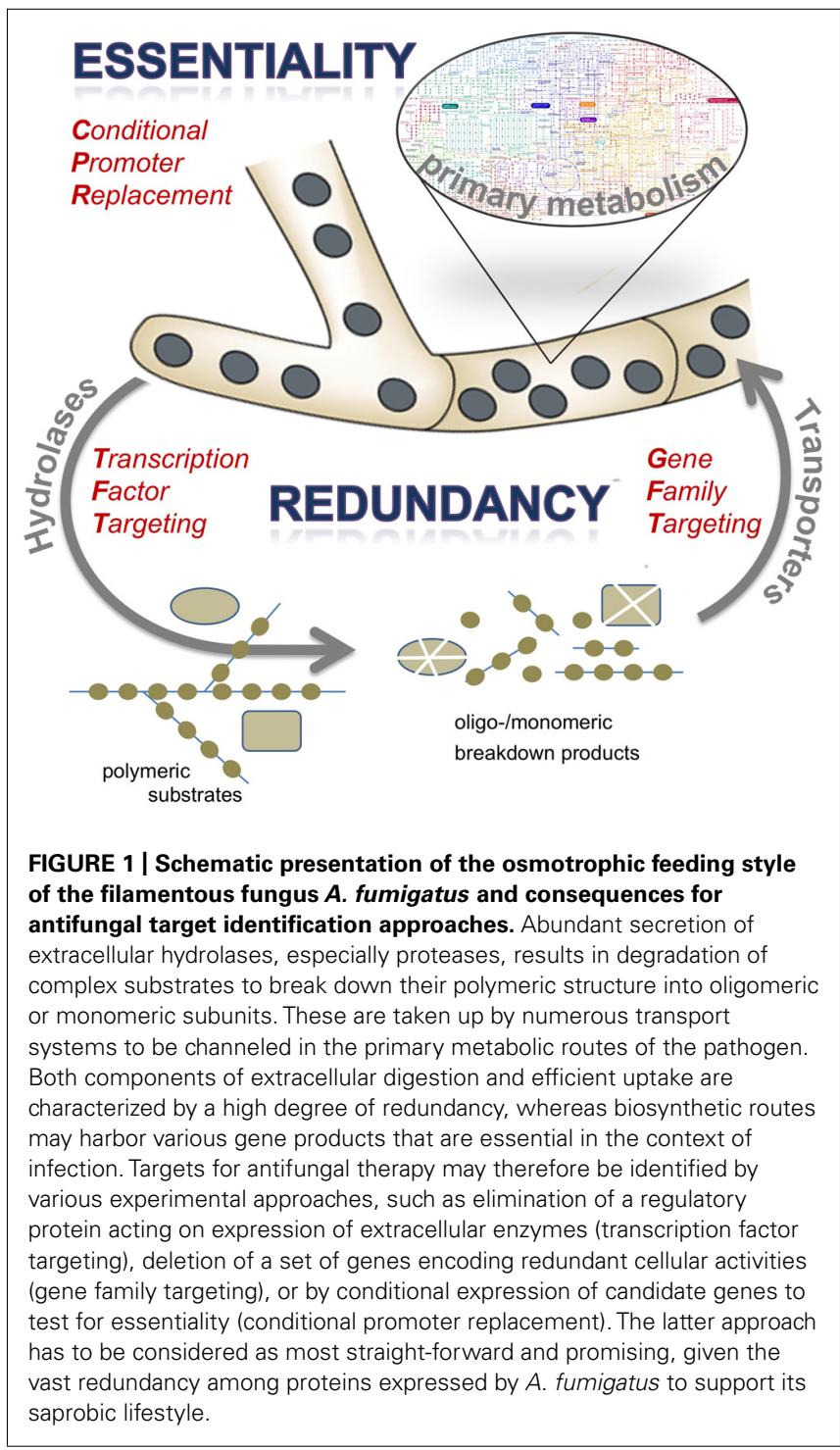

mice (McDonagh et al., 2008). By now, several additional profiling studies have sharpened our view on the transcriptional status of A. fumigatus under various environmental conditions that are relevant for pathogenesis, such as when feeding from proteinaceous substrates (Hartmann et al., 2011b), or when facing distinct stressors such as hypoxia or reactive oxidative species (McDonagh et al., 2008; Willger et al., 2008). The insights gained from such studies are instructive to identify cellular functions that are of relevance during infection and therefore assist in antifungal target identification. Validation of such promising candidates is commonly performed by approaches of reverse genetics: attenuated virulence of a corresponding A. fumigatus mutant still serves as most reliable and relevant phenotype in defining the fungal virulome for aspergillosis.

\section{TARGETING TRANSCRIPTIONAL REGULATORS}

Transcription factors are fundamental for adapting to changing conditions by modifying gene expression patterns. Consequently, 
identification and elimination of $A$. fumigatus factors necessary to cope with the host environment are an obvious option to impair its virulence. For instance it has been reported that elimination of the $z a f A$ gene encoding a zinc response transcription factor renders A. fumigatus completely avirulent in a cortisone murine model of IPA (Moreno et al., 2007). Also, elimination of the HapX transcription factor, responsible for expression of iron-uptake related genes (Hortschansky et al., 2007), causes a significant reduction of virulence in neutropenic mice (Schrettl et al., 2010). Calcium signaling through the $\mathrm{Ca}^{2+}$-binding protein calmodulin constitutes one of the most promising candidates to develop new antifungal therapy (Steinbach et al., 2006; Steinbach et al., 2011). In line with this is the transcription factor CrzA, one key target of calcineurin signaling, fundamental for virulence in a neutropenic murine model of IPA (Cramer et al., 2008; Soriani et al., 2008). In addition, the $c p c A$ gene, encoding the transcriptional effector of the crosspathway control (CPC) system (also known as general control) of amino acid biosynthesis is expressed under amino acid-limiting conditions and was shown to contribute to Aspergillus virulence (Krappmann et al., 2004; Sasse et al., 2008). However, targeting transcription factors, despite of its potential positive perspective, might not always provide a comprehensive understanding of the metabolic in vivo status. Several examples exist where targeting the master regulator of a cellular process did not result in virulence attenuation: deleting the encoding gene of the transcriptional regulator YapA resulted in strikingly reduced resistance to oxidative stress but did not impair virulence (Lessing et al., 2007; Qiao et al., 2008); a deletant for the unique transcriptional regulator PrtT turned out to be severely impaired in extracellular proteolysis and unable to utilize proteinaceous substrates but its virulence was unaffected (Bergmann et al., 2009; Sharon et al., 2009). Negative results like this indicate that either the affected cellular function does not play any role for pathogenesis in the according disease model or that redundant factors take over from the deleted ones.

\section{COPING WITH GENETIC REDUNDANCY}

Insights from recent studies on A. fumigatus' virulence yielded the fundamental conclusion that this saprobe is well-equipped to sustain its nutritional supply in the host. However, the osmotrophic lifestyle of Aspergillus, i.e., extracellular digestion of complex substrates achieved by a plethora of secreted hydrolytic enzymes (Kobayashi et al., 2007; Monod et al., 2009) and followed by uptake of breakdown products via numerous transporters, impedes a comprehensive analysis (Yoon et al., 2009). To assist in assessing any relevance of cellular functions that are encoded by multiple genes, progress was made with respect to gene targeting approaches that allows repetitive targeting of all members of a gene family to abolish any redundantly encoded cellular function (d'Enfert, 1996; Krappmann and Braus, 2003; Krappmann et al., 2005; Nielsen et al., 2006; Forment et al., 2006; Hartmann et al., 2010). By developing genetic markers, recombinase-driven excision of which can be achieved in fungo, marker rescue became feasible to allow recurring rounds of gene targeting in a straightforward manner. Depending on the number of genes to delete, such a gene family targeting approach might be worthwhile and instructive for identification of novel, fungal-specific virulence determinants. For instance, elimination of the eight-member opt gene family from the A. fumigatus genome was achieved in a pivotal study with the aim to investigate any supportive role of oligopeptide transport in pathogenesis (Hartmann et al., 2011b). The resulting octuple mutant did, however, not display any differences in virulence in comparison to its wild-type progenitor. Even interfering with extracellular proteolysis of this strain by additionally deleting the prtT gene did not alter its virulence capacities, again highlighting the pronounced degree of redundancy encoded by the A. fumigatus genome. However, this study proves that recyclable marker systems nowadays allow for straightforward repetitive gene targeting to assist in elucidating redundant features of A. fumigatus. This approach facilitates identification of relevant antifungal drug targets that are based on gene family products.

\section{BIOSYNTHETIC ROUTES AND ESSENTIAL GENES AS PRIMARY TARGETS}

The metabolic versatility displayed by A. fumigatus supports its growth and therefore virulence inside a susceptible host (Paisley et al., 2005). As described above, detailed insights in the metabolic situation was gained by in vivo transcriptional profiling studies, indicating that a pronounced starvation for nitrogen reflects the nutritional status of A. fumigatus when germinating in the lungs of neutropenic mice (McDonagh et al., 2008). In line with this, several gene products involved in nitrogen sensing and utilization had been characterized before to influence $A$. fumigatus virulence (reviewed by Krappmann and Braus, 2005). With respect to carbon, detailed analysis of a mutant strain impaired in methylcitrate cycling suggested that proteins may serve as primary source of this macroelement, a finding that is in accordance with the reported full virulence of a mutant compromised in fatty acid metabolism (Schöbel et al., 2007; Ibrahim-Granet et al., 2008). Yet, specific nutritional sources during IPA remain unidentified so far.

Despite this ignorance, several anabolic genes with their respective products have been identified to be essential for growth during pulmonary aspergillosis. In a seminal study, biosynthesis of the folate precursor para-aminobenzoic acid had been demonstrated to be strictly required for $A$. fumigatus pathogenicity in several disease models and still serves as a paradigm for an anabolic route being essential for pathogenesis (Brown et al., 2000; Slater et al., 2011). By further mutant analysis, several biosynthetic pathways had been identified to support fungal growth in the nutritionally scarce environment of murine lung tissue. One best known example is uracil biosynthesis represented the pyrG gene, which encodes orotidine- $5^{\prime}$-phosphate decarboxylase to catalyze the final step of de novo UMP biosynthesis. Mutants deleted for the pyrG gene are auxotrophs for uracil/uridine and display a severe reduction in virulence when tested in a non-neutropenic model of murine invasive aspergillosis (d'Enfert et al., 1996). As a strictly fungalspecific metabolic pathway, lysine biosynthesis turned out to be essential for full virulence, validated by infections with defined mutants deleted either for the $l y s F$ or the $h c s A$ gene (Liebmann et al., 2004; Schöbel et al., 2010). Furthermore, essentiality of the siderophore biosynthesis pathway was demonstrated by elimination of the gene sidA encoding L-ornithine- $N^{5}$-monooxygenase, which catalyzes the first committed step of hydroxamate-type siderophore biosynthesis (Schrettl et al., 2004). 
In a further development of deleting biosynthetic genes to generate auxotroph strains, pathways that are required for growth under any condition tested, and that are therefore generally essential, represent an attractive option to be selected as targets for antifungal therapy. Since the functions of the corresponding proteins are fundamental for fungal growth, their impairment would consequently eliminate virulence. An elegant approach for essential gene identification was validated by Romero et al. (2003) who developed a conditional promoter replacement (CPR) strategy further by employing the nitrogen-regulated $A$. fumigatus nii $A$ promoter (pNiiA; Romero et al., 2003; Hu et al., 2007). In this setup, expression of the gene of interest is blocked in the presence of ammonium, whereas nitrate as $\mathrm{N}$-source allows proper expression of the gene under pNiiA control. The power of this system was demonstrated by the close phenotypic correlation between pNiiA-CPR strains under repressing conditions with respective disruption mutants. Furthermore, such conditional strains could be directly tested for virulence in animal models, although being limited to systemic infections. In fact, 35 essential genes out of an initial screening pool comprising 54 candidates were identified in this seminal study, which may now be subjected to further analysis. Therefore, the CPR approach represents a valid and most straight-forward method for large scale essential gene identification.

\section{NOVEL TOOLS PROVIDE PROMISING PERSPECTIVES}

The most promising strategy for drug target prioritization to fight aspergillosis lies in the identification of non-redundant and essential gene products. As future trend emerging from distinct candidate approaches, comprehensive, large scale screening studies are reasonably required. These could include the generation of a collection of defined deletion mutants, comprising every single

\section{REFERENCES}

Abad, A., Fernandez-Molina, J. V., Bikandi, J., Ramirez, A., Margareto, J., Sendino, J., et al. (2010). What makes Aspergillus fumigatus a successful pathogen? Genes and molecules involved in invasive aspergillosis. Rev. Iberoam. Micol. 27, 155-182.

Askew, D. S. (2008). Aspergillus fumigatus: virulence genes in a street-smart mold. Curr. Opin. Microbiol. 11, 331-337.

Baddley, J. W., Andes, D. R., Marr, K. A., Kontoyiannis, D. P., Alexander, B. D., Kauffman, C. A., et al. (2010). Factors associated with mortality in transplant patients with invasive aspergillosis. Clin. Infect. Dis. 50, 1559-1567.

Bergmann, A., Hartmann, T., Cairns, T., Bignell, E. M., and Krappmann, S. (2009). A regulator of Aspergillus fumigatus extracellular proteolytic activity is dispensable for virulence. Infect. Immun. 77, 40414050.

Bhabhra, R., and Askew, D. S (2005). Thermotolerance and virulence of Aspergillus fumigatus: role of the fungal nucleolus. Med. Mycol. 43(Suppl. 1), S87-S93.

Brakhage, A. A., and Langfelder, K. (2002). Menacing mold: the molecular biology of Aspergillus fumigatus. Annu. Rev. Microbiol. 56, 433-455.

Brock, M. (2009). Fungal metabolism in host niches. Curr. Opin. Microbiol. 12, 371-376.

Brown, J. S., Aufauvre-Brown, A., Brown, J., Jennings, J. M., Arst, H. Jr., and Holden, D. W. (2000). Signature-tagged and directed mutagenesis identify PABA synthetase as essential for Aspergillus fumigatus pathogenicity. Mol. Microbiol. 36 1371-1380.

Casadevall, A., and Pirofski, L. A. (1999). Host-pathogen interactions: redefining the basic concepts of virulence and pathogenicity. Infect. Immun. 67, 3703-3713.

Cramer, R. A. Jr., Perfect, B. Z., Pinchai, N., Park, S., Perlin, D. S., Asfaw, Y. G., et al. (2008). Calcineurin target CrzA regulates conidial germination, hyphal growth, and pathogenesis of Aspergillus fumigatus. Eukaryot. Cell 7, 1085-1097.

gene annotated from a refined $A$. fumigatus genome sequence. Including conditional alleles would further scrutinize essential cellular functions that are based on single gene products and appears as a superior strategy. In particular, recent developments that demonstrated the feasibility of doxycycline-dependent expression modules in Aspergillus (Vogt et al., 2005; Meyer et al., 2011; Dichtl et al., 2012) pave the road for such advanced CPR studies that might even allow for virulence tests in pulmonary aspergillosis models. As an alternative to extensive and randomized screening, network approaches of systems biology might serve as more efficient and straight-forward tactic (Chavali et al., 2012; Horn et al., 2012). In conjunction, new molecular tools for multiple gene targeting will allow comprehensive understanding of the in vivo A. fumigatus metabolic status. Concluding, the advanced molecular biology of A. fumigatus (Krappmann, 2006) holds the future promise of innovative strategies for antifungal drug target identification, an urgent task in fighting against this first mould pathogen in Europe (Senior, 2009).

\section{ACKNOWLEDGMENTS}

We are indebted to the European Science Foundation for support within the Fuminomics Research Networking Programme (06RNP-132: "Functional genomics in Aspergillus fumigatus and new strategies to fight against the first fungal pathogen in Europe") and all other members of this framework for continuous discussion and inspiration. The helpful assistance from members of YIG3 and the Institute of Molecular Infection Biology is highly acknowledged, as is the financial support by the German Research Foundation (KR2294/1), the Research Center for Infectious Diseases, the University of Würzburg, the Free State of Bavaria, and the University Hospital of Erlangen by its Microbiology Institute.

Chavali, A. K., D’Auria, K. M., Hewlett, E. L., Pearson, R. D., and Papin, J. A. (2012). A metabolic network approach for the identification and prioritization of antimicrobial drug targets. Trends Microbiol. 20, 113-123.

Cutler, J. E. (1991). Putative virulence factors of Candida albicans. Annu. Rev. Microbiol. 45, 187-218.

d'Enfert, C. (1996). Selection of multiple disruption events in Aspergillus fumigatus using the orotidine- $5^{\prime}$ decarboxylase gene, $p y r G$, as unique transformation marker. Curr. Genet. 30, 76-82

d'Enfert, C., Diaquin, M., Delit, A., Wuscher, N., Debeaupuis, J. P., Huerre, M., et al. (1996). Attenuated virulence of uridine-uracil auxotrophs of Aspergillus fumigatus. Infect. Immun. 64, 4401-4405.

Del Bono, V., Mikulska, M., and Viscoli, C. (2008). Invasive aspergillosis: diagnosis, prophylaxis and treatment. Curr. Opin. Hematol. 15, 586-593.

Dichtl, K., Helmschrott, C., Dirr, F., and Wagener, J. (2012). Deciphering cell wall integrity signalling in
Aspergillus fumigatus: identification and functional characterization of cell wall stress sensors and relevant Rho GTPases. Mol. Microbiol. 83, 506-519.

Forment, J.V., Ramón, D., and MacCabe A. P. (2006). Consecutive gene deletions in Aspergillus nidulans: application of the Cre/loxP system. Curr. Genet. 50, 217-224.

Fuller, K. K., Richie, D. L., Feng, X., Krishnan, K., Stephens, T. J., Wikenheiser-Brokamp, K. A., et al. (2011). Divergent protein kinase A isoforms co-ordinately regulate conidial germination, carbohydrate metabolism and virulence in Aspergillus fumigatus. Mol. Microbiol. 79, 1045-1062.

Grahl, N., Puttikamonkul, S., Macdonald, J. M., Gamcsik, M. P., Ngo, L. Y., Hohl, T. M., et al. (2011). In vivo hypoxia and a fungal alcohol dehydrogenase influence the pathogenesis of invasive pulmonary aspergillosis. PLoS Pathog. 7, e1002145. doi: 10.1371/journal.ppat.1002145

Haas, H. (2012). Iron - a key nexus in the virulence of Aspergillus 
fumigatus. Front. Microbiol. 3:28. doi: 10.3389/fmicb. 2012.00028

Hartmann, T., Sasse, C., Schedler, A., Hasenberg, M., Gunzer, M., and Krappmann, S. (2011a). Shaping the fungal adaptome - stress responses of Aspergillus fumigatus. Int. J. Med. Microbiol. 301, 408-416.

Hartmann, T., Cairns, T. C., Olbermann, P., Morschhäuser, J., Bignell, E. M., and Krappmann, S. (2011b) Oligopeptide transport and regulation of extracellular proteolysis are required for growth of Aspergillus fumigatus on complex substrates but not for virulence. Mol. Microbiol. 82, 917-935.

Hartmann, T., Dumig, M., Jaber, B. M., Szewczyk, E., Olbermann, P., Morschhäuser, J., et al. (2010). Validation of a self-excising marker in the human pathogen Aspergillus fumigatus by employing the $\beta$-rec/six site-specific recombination system. Appl. Environ. Microbiol. 76, 63136317.

Hoehamer, C. F., Cummings, E. D., Hilliard, G. M., and Rogers, P. D. (2010). Changes in the proteome of Candida albicans in response to azole, polyene, and echinocandin antifungal agents. Antimicrob. Agents Chemother. 54, 1655-1664.

Horn, F., Heinekamp, T., Kniemeyer, O., Pollmacher, J., Valiante, V., and Brakhage, A. A. (2012). Systems biology of fungal infection. Front. Microbiol. 3:108. doi 10.3389/fmicb.2012.00108

Hortschansky, P., Eisendle, M., AlAbdallah, Q., Schmidt, A. D. Bergmann, S., Thon, M., et al. (2007). Interaction of HapX with the CCAAT-binding complex - a novel mechanism of gene regulation by iron. EMBO J. 26, 3157-3168.

Hu, W., Sillaots, S., Lemieux, S., Davison, J., Kauffman, S., Breton, A. et al. (2007). Essential gene identification and drug target prioritization in Aspergillus fumigatus. PLoS Pathog. 3, e24. doi: 10.1371/journal.ppat.0030024

Hube, B. (2004). From commensal to pathogen: stage- and tissue-specific gene expression of Candida albicans. Curr. Opin. Microbiol. 7, 336-341.

Ibrahim-Granet, O., Dubourdeau, M., Latgé, J.-P., Ave, P., Huerre, M., Brakhage, A. A., et al. (2008). Methylcitrate synthase from Aspergillus fumigatus is essential for manifestation of invasive aspergillosis. Cell. Microbiol. 10, 134-148.

Jung, W. H., Sham, A., Lian, T., Singh, A., Kosman, D. J., and Kronstad, J. W. (2008). Iron source preference and regulation of iron uptake in Cryptococcus neoformans. PLoS Pathog. 4, e45. doi: 10.1371/journal.ppat.0040045

Kobayashi, T., Abe, K., Asai, K. Gomi, K., Juvvadi, P. R., Kato, M., et al. (2007). Genomics of Aspergillus oryzae. Biosci. Biotechnol. Biochem. 72, 646-670.

Krappmann, S. (2006). Tools to study molecular mechanisms of Aspergillus pathogenicity. Trends Microbiol. 14, 356-364.

Krappmann, S., Bayram, Ö., and Braus, G. H. (2005). Deletion and allelic exchange of the Aspergillus fumiga tus veA locus via a novel recyclable marker module. Eukaryot. Cell 4 1298-1307.

Krappmann, S., Bignell, E. M., Reichard, U., Rogers, T., Haynes, K., and Braus, G. H. (2004). The Aspergillus fumigatus transcriptional activator $\mathrm{CpcA}$ contributes significantly to the virulence of this fungal pathogen. Mol. Microbiol. 52, 785-799.

Krappmann, S., and Braus, G. H. (2003). Deletion of Aspergillus nidulans aro $C$ using a novel blaster module that combines ET cloning and marker rescue. Mol. Genet. Genomics 268, 675-683.

Krappmann, S., and Braus, G. H. (2005). Nitrogen metabolism of Aspergillus and its role in pathogenicity. Med. Mycol. 43(Suppl. 1) S31-S40.

Latgé, J.-P. (1999). Aspergillus fumigatus and aspergillosis. Clin. Microbiol. Rev. 12, 310-350.

Latgé, J.-P. (2001). The pathobiology of Aspergillus fumigatus. Trends Microbiol. 9, 382-389.

Lessing, F., Kniemeyer, O., Wozniok, I., Loeffler, J., Kurzai, O., Härtl, A., et al. (2007). The Aspergillus fumigatus transcriptional regulator AfYap1 represents the major regulator for defense against reactive oxygen intermediates but is dispensable for pathogenicity in an intranasal mouse infection model. Eukaryot. Cell 6, 2290-2302.

Liebmann, B., Muhleisen, T. W., Muller, M., Hecht, M., Weidner, G., Braun, A., et al. (2004). Deletion of the Aspergillus fumigatus lysine biosynthesis gene lysF encoding homoaconitase leads to attenuated virulence in a low-dose mouse infection model of invasive aspergillosis. Arch. Microbiol. 181, 378-383.

McDonagh, A., Fedorova, N. D., Crabtree, J., Yu, Y., Kim, S., Chen, D., et al. (2008). Sub-telomere directed gene expression during initiation of invasive aspergillosis. PLoS Pathog. 4, e1000154. doi: 10.1371/journal.ppat. 1000154
McNeil, M. M., Nash, S. L., Hajjeh, R. A. Phelan, M. A., Conn, L. A., Plikaytis B. D., et al. (2001). Trends in mortality due to invasive mycotic diseases in the United States, 1980-1997. Clin. Infect. Dis. 33, 641-647.

Meyer, V., Wanka, F., van Gent, J., Arentshorst, M., van den Hondel, C. A., and Ram, A. F. (2011). Fungal gene expression on demand: an inducible, tunable, and metabolismindependent expression system for Aspergillus niger. Appl. Environ. Microbiol. 77, 2975-2983.

Mikulska, M., Raiola, A. M., Bruno, B., Furfaro, E., Van Lint, M. T., Bregante, S., et al. (2009). Risk factors for invasive aspergillosis and related mortality in recipients of allogeneic SCT from alternative donors: an analysis of 306 patients. Bone Marrow Transplant. 44, 361-370.

Monod, M., Jousson, O., and Reichard, U. (2009). "Aspergillus fumigatus secreted proteases," in Aspergillus fumigatus and Aspergillosis, eds. J.-P. Latgé and W. J. Steinbach (Washington, DC: ASM Press), 87-106.

Moreno, M. A., Ibrahim-Granet, O., Vicentefranqueira, R., Amich, J., Ave, P., Leal, F., et al. (2007). The regulation of zinc homeostasis by the ZafA transcriptional activator is essential for Aspergillus fumigatus virulence. Mol. Microbiol. 64, 1182-1197.

Nielsen, M. L., Albertsen, L., Lettier, G. Nielsen, J. B., and Mortensen, U. H. (2006). Efficient PCR-based gene targeting with a recyclable marker for Aspergillus nidulans. Fungal Genet. Biol. 43, 54-64.

Nierman, W. C., Pain, A., Anderson, M. J., Wortman, J. R., Kim, H. S., Arroyo, J., et al.(2005). Genomic sequence of the pathogenic and allergenic filamentous fungus Aspergillus fumigatus. Nature 438, 11511156.

Paisley, D., Robson, G. D., and Denning, D. W. (2005). Correlation between in vitro growth rate and in vivo virulence in Aspergillus fumigatus. Med. Mycol. 43, 397-401.

Qiao, J., Kontoyiannis, D. P., Calderone, R., Li, D., Ma, Y., Wan, Z., et al. (2008). Afyap 1, encoding a bZip transcriptional factor of Aspergillus fumigatus, contributes to oxidative stres response but is not essential to the virulence of this pathogen in mice immunosuppressed by cyclophosphamide and triamcinolone. Med. Mycol. 46, 773-782.

Ramanan, N., and Wang, Y. (2000). A high-affinity iron permease essential for Candida albicans virulence. Science 288, 1062-1064.
Rhodes, J. C. (2006). Aspergillus fumigatus: growth and virulence. Med. Mycol. 44, S77-S81.

Romero, B., Turner, G., Olivas, I., Laborda, F., and De Lucas, J. R. (2003). The Aspergillus nidulans alcA promoter drives tightly regulated conditional gene expression in Aspergillus fumigatus permitting validation of essential genes in this human pathogen. Fungal Genet. Biol. 40, 103-114.

Sasse, C., Bignell, E. M., Hasenberg, M., Haynes, K., Gunzer, M., Braus, G. H., et al. (2008). Basal expression of the Aspergillus fumigatus transcriptional activator CpcA is sufficient to support pulmonary aspergillosis. Fungal Genet. Biol. 45, 693-704.

Schöbel, F., Ibrahim-Granet, O., Ave, P., Latgé, J.-P., Brakhage, A. A., and Brock, M. (2007). Aspergillus fumigatus does not require fatty acid metabolism via isocitrate lyase for development of invasive aspergillosis. Infect. Immun. 75, 1237-1244.

Schöbel, F., Jacobsen, I. D., and Brock, M. (2010). Evaluation of lysine biosynthesis as an antifungal drug target: biochemical characterization of Aspergillus fumigatus homocitrate synthase and virulence studies. Eukaryot. Cell 9, 878-893.

Schrettl, M., Beckmann, N., Varga, J., Heinekamp, T., Jacobsen, I. D., Jochl, C., et al. (2010). HapX-mediated adaption to iron starvation is crucial for virulence of Aspergillus fumigatus. PLoS Pathog. 6, e1001124. doi: 10.1371/journal.ppat.1001124

Schrettl, M., Bignell, E., Kragl, C., Jöchl, C., Rogers, T., Arst, H. N. Jr., et al. (2004). Siderophore biosynthesis but not reductive iron assimilation is essential for Aspergillus fumigatus virulence. J. Exp. Med. 200, 12131219.

Senior, K. (2009). Genomics to target fungal lung disease. Lancet Infect. Dis. 8, 667 .

Sharon, H., Hagag, S., and Osherov, N. (2009). Transcription factor PrtT controls expression of multiple secreted proteases in the human pathogenic mold Aspergillus fumigatus. Infect. Immun. 77, 4051-4060.

Singh, N., and Paterson, D. L. (2005). Aspergillus infections in transplant recipients. Clin. Microbiol. Rev. 18, 44-69.

Slater, J. L., Gregson, L., Denning, D. W., and Warn, P. A. (2011). Pathogenicity of Aspergillus fumigatus mutants assessed in Galleria mellonella matches that in mice. Med. Mycol. 49(Suppl. 1), S107-S113. 
Soriani, F. M., Malavazi, I., Da Silva Ferreira, M. E., Savoldi, M., von Zeska Kress, M. R., De Souza Goldman, M. H., et al. (2008). Functional characterization of the Aspergillus fumigatus CRZ1 homologue, CrzA. Mol. Microbiol. 67, 1274-1291.

Sugui, J. A., Kim, H. S., Zarember, K. A., Chang, Y. C., Gallin, J. I., Nierman, W. C., et al. (2008). Genes differentially expressed in conidia and hyphae of Aspergillus fumigatus upon exposure to human neutrophils. PLoS ONE 3, e2655. doi: 10.1371/journal. pone. 0002655

Steinbach, W. J., Cramer, R. A. Jr., Perfect, B. Z., Asfaw, Y. G., Sauer, T. C., Najvar, L. K., et al. (2006). Calcineurin controls growth, morphology, and pathogenicity in Aspergillus fumigatus. Eukaryot. Cell 5, 10911103.

Steinbach, W. J., Juvvadi, P. R., Fortwendel, J. R., and Rogg, L. E. (2011).
Newer combination antifungal therapies for invasive aspergillosis. Med. Mycol. 49(Suppl. 1), S77-S81.

Tekaia, F., and Latgé, J.-P. (2005). Aspergillus fumigatus: saprophyte or pathogen? Curr. Opin. Microbiol. 8, 385-392.

van Burik, J. A., and Magee, P. T. (2001) Aspects of fungal pathogenesis in humans. Annu. Rev. Microbiol. 55 743-772.

Vogt, K., Bhabhra, R., Rhodes, J. C., and Askew, D. S. (2005). Doxycycline-regulated gene expression in the opportunistic fungal pathogen Aspergillus fumigatus. BMC Microbiol. 5, 1. doi: 10.1186/1471 2180-5-1

Wang, W. W. X., Liu, J., Masaharu, I., Yasuo, I., and Cui, Z. (2007). Effect of oxygen concentration on the composting process and maturity. Compost Sci. Util. 15, 184-190.
Willger, S. D., Puttikamonkul, S. Kim, K. H., Burritt, J. B., Grahl, N., Metzler, L. J., et al. (2008) A sterol-regulatory element binding protein is required for cell polarity, hypoxia adaptation, azole drug resistance, and virulence in Aspergillus fumigatus. PLoS Pathog. 4 e1000200. doi: 10.1371/journal.ppat. 1000200

Yoon, J., Kimura, S., Maruyama, J. and Kitamoto, K. (2009). Construction of quintuple protease gene disruptant for heterologous protein production in Aspergillus oryzae. Appl. Microbiol. Biotechnol. 82, 691-701.

Conflict of Interest Statement: The authors declare that the research was conducted in the absence of any commercial or financial relationships that could be construed as a potential conflict of interest.

Received: 21 August 2012; accepted: 15 November 2012; published online: 03 December 2012.

Citation: Amich $J$ and Krappmann $S$ (2012) Deciphering metabolic traits of the fungal pathogen Aspergillus fumigatus: redundancy vs. essentiality. Front. Microbio. 3:414. doi: 10.3389/fmicb.2012. 00414

This article was submitted to Frontiers in Fungi and Their Interactions, a specialty of Frontiers in Microbiology.

Copyright (c) 2012 Amich and Krappmann. This is an open-access article distributed under the terms of the Creative Commons Attribution License, which permits use, distribution and reproduction in other forums, provided the original authors and source are credited and subject to any copyright notices concerning any third-party graphics etc. 